\title{
Facial somatosensorial evaluation in idiopathic trigeminal neuralgia
}

\author{
Avaliação somatosensitiva facial em neuralgia idiopática do trigêmeo \\ Luciana Alvarenga da Silva1, Silvia Regina Dowgan Tesseroli de Siqueira², José Tadeu Tesseroli de Siqueira ${ }^{3}$, \\ Manoel Jacobsen Teixeira ${ }^{4}$
}

Idiopathic trigeminal neuralgia (ITN) is identified by paroxysmal unilateral shock-like pain, distributed in one or more trigeminal branches, with clear diagnostic criteria based on history and clinical exams. Etiology and physiopathology remain obscure. Recently, abnormal expression of voltage-gated sodium channels had been described in these patients, suggesting that ITN could be a channelopathy ${ }^{1}$.

Current diagnostic criteria do not include sensorial deficit; however, recent studies have shown that discrete abnormalities may be present in trigeminal territories ${ }^{2}$, which could be associated with the chronic evolution of such pain. There are no studies investigating patients nearly onset.

A 64-year old, male, was diagnosed with ITN according to the criteria of the International Association for the Study of Pain (IASP) ${ }^{3}$. He was evaluated with a systematized protocol of facial sensitivity, which included the clinical exam and quantitative sensory testing: gustative thresholds, olfactory thresholds, quantitative salivary and somatosensorial evaluation; all trigeminal branches (front, cheek, and chin) were also evaluated bilaterally: thermal detection, mechanical detection, vibration detection, electric detection, and pain detection threshold.

The patient did not have abnormalities in imaging exams (computed tomography or magnetic resonance) or any neurological deficits in the evaluation by the neurologist. Pain had started in the last two weeks. Until the moment of evaluation, the patient had not been prescribed for ITN and was not using any medication.

There was a high sweet threshold and absence of identification of the salty taste. Mean salivary flow was $0.2363 \mathrm{~g} / \mathrm{min}$. There were differences between the facial sides in somatosensory thresholds: warm, mechanical, vibration, electric and pain (Figure).
These thresholds were higher at the pain side (right), when compared to the left one at the three trigeminal branches, except for the cold threshold, which was lower at the pain than the opposite side. After the evaluation, carbamazepine was prescribed (400 mg/day) and after one week there was complete relieve of symptoms. These data suggest evidence for future studies, which support neuropathic mechanisms in ITN $^{1}$, and the need to investigate patients in the early onset in order to verify the prevalence of these findings. Besides, there was a reduced gustative detection for some tastes, especially salty, which had already been described for ITN $^{4}$ and supports central sensitization involved in the physiopathology of this disease since the beginning of onset. There is also in the literature the description of gustative stimuli as triggering factors for ITN in some patients ${ }^{5}$ and the role of that must be understood.

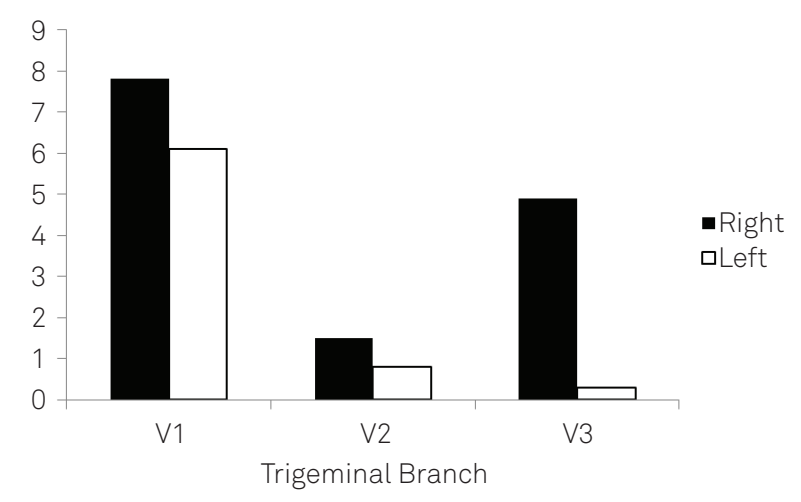

Pain threshold

*V1: ophthalmic branch; V2: maxillary branch; V3: mandibular branch Figure. Pain thresholds at the three trigeminal branches: comparison between facial sides.

\footnotetext{
Neurology Department, Medical School, University of São Paulo (USP), São Paulo SP, Brazil;

${ }^{1}$ Gerontologist, Postgraduation student, Neurology Department, Medical School, USP, São Paulo SP, Brazil;

${ }^{2}$ PhD, DDS, Assistant Professor of Gerontology, School of Arts, Science and Humanities, USP, São Paulo SP, Brazil;

${ }^{3}$ PhD, DDS, Head of the Orofacial Pain Team, Dentistry Division, Hospital das Clínicas, Medical School, USP, São Paulo SP, Brazil;

${ }^{4}$ PhD, MD, Full Professor of Neurosurgery, Neurology Division, Medical School, USP, São Paulo SP, Brazil.

Correspondence: Luciana Alvarenga da Silva; Alameda Leningrado, 204; 06437-000Barueri SP - Brasil; E-mail: lucianaa@usp.br

Support: This research was supported by the Orofacial Pain Team of the Hospital das Clínicas, Medical School, University of São Paulo, Brazil.

Conflict of interest: There is no conflict of interest to declare.

Received 21 June 2011; Received in final form 02 August 2011; Accepted 09 August 2011
} 
In conclusion, this study presented a case of ITN with early onset. Sensory abnormalities were observed at the affected side when compared to the opposite facial one in all trigeminal branches; this should be investigated in larger samples in order to better understand the physiopathology of this disease, which remains obscure.

\section{References}

1. Siqueira SR, Alves B, Malpartida HM, Teixeira MJ, Siqueira JT. Abnormal expression of voltage-gated sodium channels Nav1.7, Nav1.3 and Nav1.8 in trigeminal neuralgia. Neurosciences 2009;164:573-577

2. Synai VJ, Bonamico LH, Dubrovsky A. Subclinical abnormalities in trigeminal neuralgia. Cephalalgia 2003;23:541-544

3. Merskey H, Bogduk N. Classification of chronic pain: descriptions of chronic pain syndromes and definitions of painterms. Seattle IASP Press; 1994.

4. Siviero M, Teixeira MJ, de Siqueira JT, Siqueira SR. Somesthetic, gustatory, olfactory function and salivary flow in patients with neuropathic trigeminal pain. Oral Dis 2010;16:482-487.

5. Sharav Y, Benoliel R, Schnarch A, Greenberg L. Idiopathic trigeminal pain associated with gustatory stimuli. Pain 1991;44:171-174. 